\title{
Soluble thrombomodulin concentration is raised in scleroderma associated pulmonary hypertension
}

\author{
Richard J Stratton, Lucie Pompon, John G Coghlan, Jeremy D Pearson, Carol M Black
}

\begin{abstract}
Objective-To investigate the expression of thrombomodulin in scleroderma associated pulmonary hypertension. Methods-Soluble thrombomodulin (sTM), was measured in plasma samples from 34 scleroderma patients shown to have pulmonary hypertension at echocardiogram, and comparison drawn against samples from 38 scleroderma control patients, and 20 healthy controls. Serial measurements of sTM were performed in the 34 patients with scleroderma associated pulmonary hypertension to investigate possible changes in STM concentration with progression of the condition.
\end{abstract}

Results-Mean sTM was raised in scleroderma associated pulmonary hypertension when compared with scleroderma controls (mean sTM $65.4 \mathrm{ng} / \mathrm{ml} \quad v \quad 43.3$ $\mathrm{ng} / \mathrm{ml}, \mathrm{p}<0.05)$, and when compared with healthy controls (mean sTM $38.1 \mathrm{ng} / \mathrm{ml}$, $p<0.05)$. There was no significant difference between mean sTM in scleroderma controls and healthy controls. Mean STM concentration did not change with progression of pulmonary hypertension.

Conclusion-Plasma sTM is raised in scleroderma associated pulmonary hypertension. The pathogenesis of scleroderma associated pulmonary hypertension may be distinct from the pathogenesis of other forms of pulmonary vascular disease. (Ann Rheum Dis 2000;59:132-134)

A form of pulmonary vascular disease, characterised by intimal proliferation and concentric narrowing of pulmonary arteries of all sizes, is a common postmortem finding in scleroderma. ${ }^{1}$ The presence of these lesions correlates with a clinical picture of progressively severe pulmonary hypertension culminating in death through circulatory failure. ${ }^{2}$ The pathogenesis of scleroderma associated pulmonary hypertension is not understood.

Accepted for publication 1 October 1999
Thrombomodulin (TM) is an anticoagulant glycoprotein expressed by endothelial cells. TM binds thrombin and changes its substrate specificity, so that thrombin then activates protein C. ${ }^{3}$ Thrombomodulin expression is of interest in scleroderma associated pulmonary hypertension for a number of reasons. Thrombosis of pulmonary arteries is an important factor in the development of severe pulmonary hypertension. ${ }^{4}$ One possibile explanation for this observation is that in pulmonary hypertension the pulmonary vascular endothelium is deficient in anticoagulant proteins like TM. Also injury to endothelial cells might contribute in the pathogenesis of scleroderma associated pulmonary hypertension and plasma soluble TM (sTM) is increased in conditions associated with endothelial cell damage. ${ }^{5}$ In this study comparison has been made between the mean plasma sTM concentration of patients with scleroderma associated pulmonary hypertension, a scleroderma control population, and a healthy control population.

\section{Methods}

Scleroderma was diagnosed according to the preliminary criteria of the American Rheumatology Association. ${ }^{6}$ Patients were selected from a database of over 800 scleroderma patients. Mean systolic pulmonary artery pressure was determined by annual Doppler echocardiogram. Blood samples were taken on the morning of the procedure and stored at $-40^{\circ} \mathrm{C}$ before analysis. The onset of pulmonary hypertension was defined by a mean systolic pulmonary artery pressure of greater than $30 \mathrm{~mm} \mathrm{Hg}$ at echocardiogram.

Patients with raised plasma creatinine were excluded from the study as STM is cleared by the renal route. Patients were included as follows; scleroderma associated pulmonary hypertension 34 patients, scleroderma controls 38 patients, healthy controls 20 (baseline characteristics in table 1 ).

The possibility that plasma sTM concentration changes as the severity of pulmonary hypertension increases was investigated in the 34 patients with scleroderma associated pulmonary hypertension using plasma samples (three or five samples per patient) collected at six month intervals after the diagnosis of pulmonary hypertension with synchronous pulmonary artery pressure measurements by echocardiogram.

Plasma sTM was measured by a commercial enzyme linked immunoassay (Diagnostica Stago, Asnieres-sur-Seine, France). 


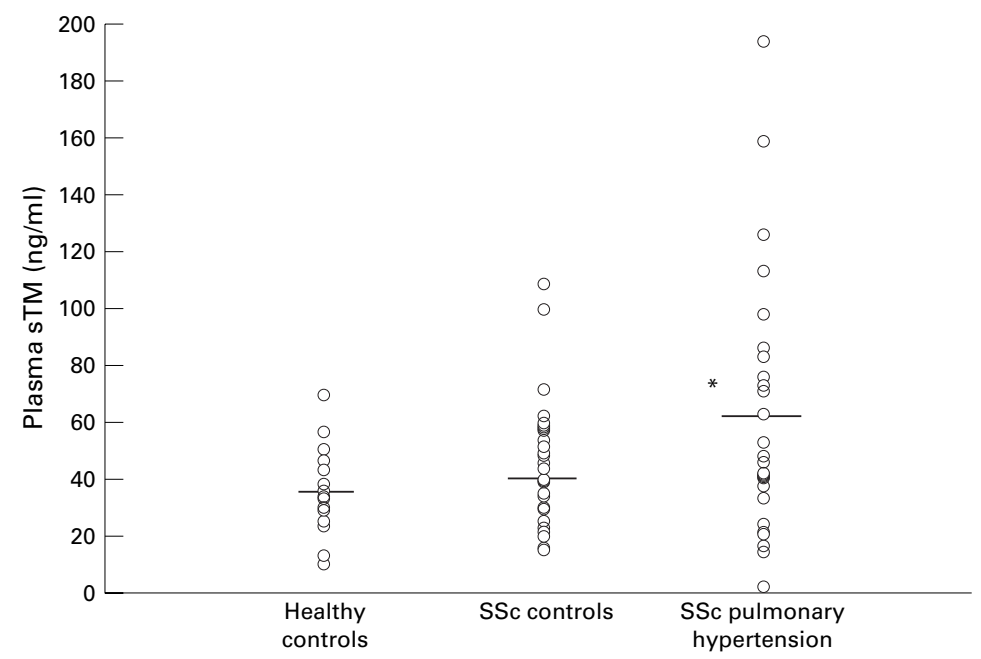

Figure 1 Plasma soluble thrombomodulin concentration against patient group. Solid bars indicated mean soluble thrombomodulin concentration. ${ }^{\star} p<0.05$ versus scleroderma and healthy control values. sponding baseline values using a paired Student's $t$ test. Bonferroni's correction was applied to correct for the multiple application of the paired $t$ test. A similar method was used to compare sequential measurements of systolic pulmonary artery pressure against baseline measurements.

\section{Results}

There was a significant difference between the means of the three groups studied (one way analysis of variance test, $F$ 7.46, $\mathrm{p}=0.001)$. Mean plasma sTM concentration was raised in plasma from patients with scleroderma associated pulmonary hypertension when compared with scleroderma controls (mean (SEM) sTM 65.4 (7.0) $\mathrm{ng} / \mathrm{ml}$, versus 43.3 (3.4) $\mathrm{ng} / \mathrm{ml}$, $\mathrm{p}<0.05)$ and when compared with healthy controls (mean sTM 38.1 (3.4) ng/ml, p<0.05) (fig 1). Mean sTM concentrations did not differ between scleroderma control and healthy control populations.

In the 34 patients with scleroderma associated pulmonary hypertension severity of pulmonary hypertension increased over a 24 month follow up period from a mean (SEM) systolic pulmonary artery pressure of $36 \mathrm{~mm}$ $\mathrm{Hg}$ (3.0) at baseline to a mean of 52.3 (5.7) $\mathrm{mm} \mathrm{Hg}$ at 24 months $(\mathrm{p}<0.001)$. Over this period there was no significant change in STM concentration (fig 2).

\section{Discussion}

Release of sTM by cultured endothelial cells is increased by conditions favouring endothelial cell death, ${ }^{8}$ and in vivo increased sTM is found in conditions where there is widespread damage to the vascular endothelium. ${ }^{5}$ In this study we have shown that STM is raised in the plasma of patients with scleroderma associated pulmonary hypertension but not in plasma from scleroderma control patients. Endothelial cell injury is an important mechanism in the development of scleroderma. ${ }^{9}$ Damage to pulmonary vascular endothelial cells could account for the increase of sTM found in this study.

Both increased shear stress and hypoxic conditions lead to decreased expression of TM by endothelial cells in vitro. ${ }^{10} 11$ Previous studies show that plasma sTM concentrations are decreased in patients with severe idiopathic primary pulmonary hypertension. ${ }^{12}{ }^{13}$ Therefore it was suggested that sTM might decrease with increasing severity of scleroderma associated pulmonary hypertension. In this study, however, we found no significant change in sTM concentration as pulmonary hypertension worsened over a two year follow up. Possibly the mechanisms leading to scleroderma associated pulmonary hypertension are different from those leading to other forms of pulmonary vascular disease such as idiopathic primary pulmonary hypertension.

This study adds emphasis to one further point. Early pathogenic mechanisms in pulmonary hypertension can be studied in scleroderma as it is practical to screen the scleroderma population for the development of this complication. Other forms of pulmonary vascular disease such
Figure 2 Progression of pulmonary hypertension over a two year period in 34 patients with scleroderma associated pulmonary hypertension. ${ }^{\star} p<0.05,{ }^{\star} * 0<0.001$ versus baseline measurement of pulmonary artery pressure. Serial measurements of sTM showing no significant change over two year follow up. 
as idiopthic primary pulmonary hypertension cannot usually be diagnosed at an early asymptomatic stage as the at risk population cannot be clearly identified.

Funding: this study was supported by a fellowship from the Ernst Schering foundation.

1 D'Angelo WA, Fries JF, Masi AT, Shulman LE. Pathologic observations in systemic sclerosis. A study of fifty eight autopsy cases and fifty eight matched controls. Am J Med 1969;46:428-40.

2 Stupi AM, Steen VD, Owens GR, Barnes EL, Rodnan GP, Medsger TA Jr. Pulmonary hypertension in the CREST Medser TA Jr. Pu Of 1986;29:515-24

3 Dittman WA, Majerus PW. Structure and function of thrombomodulin: a natural anticoagulant. Blood 1990;75: 329-36.

4 Chaouat A, Weitzenblum E, Higgenbottam T. The role of thrombosis in severe pulmonary hypertension. Eur Resp J 1996;9:356-63.

5 Mercie P, Seigneur M, Constans J, Boisseau M, Conri C. Assay of thrombomodulin in systemic diseases. Rev Med Interne 1997;18:126-31.
6 Subcommittee for scleroderma criteria of the american rheumatism association diagnostic and therapeutic criteria committee. Preliminary criteria for the classification of sysemic sclerosis (scleroderma). Arthritis Rheum 1980;23: $581-90$

7 Scheffe H. The analysis of variance. New York: Wiley, 1959.

8 Ishii $\mathrm{H}$, Uchiyama $\mathrm{H}$, Kazama M. Soluble thrombomodulin antigen in conditioned medium is increased by damage of endothelial cells Thromb Haemost 1991;65:618-23.

9 Pearson JD. The endothelium: its role in scleroderma. Ann Rheum Dis 1991;50:866-71.

10 Malek AM, Jackman R, Rosenberg RD, Izumo S. Endotheial expression of thrombomodulin is reversibly regulated by fluid shear stress. Clin Res 1994;74:852-60.

11 Shreeniwas R, Ogawa S, Cozzolino F, Torcia G, Braunstein $\mathrm{N}$, Butura $\mathrm{C}$, et al. Macrovascular and microvascular endothelium during long term hypoxia: alterations in cell growth, monolayer permeability, and cell surface coagulant properties J Cell Physiol 1991;146:8-17.

12 Cacoub P, Karmochkine M, Dorent R, Nataf P, Piette JC, Godeu P, et al. Plasma levels of thrombomodulin in pulmonary hypertension. Am J Med 1996;101:160-4.

13 Welsh CH, Hassell KL, Badesh DB, Kressin DC, Marlar RA. Coagulation and fibrinolytic profiles in patients with severe pulmonary hypertension. Chest 1996; 110:710-17. 